\title{
Comparative outcomes between palliative ileostomy and colostomy in patients with malignant large bowel obstruction
}

\author{
Yasuo Nagashima, Kimihiko Funahashi, Mitsunori Ushigome, Satoru Kagami, Tomoaki Kaneko, Yu Yoshino, \\ Takamaru Koda, Junichi Koike, Akiharu Kurihara, Takamitsu Sakai and Mayu Goto \\ Department of General and Gastroenterological Surgery, Toho University Omori Medical Center, Tokyo, Japan
}

\begin{abstract}
:
Objectives: Palliative stoma creation should be considered in patients at high risk of colonic metallic stent failure. However, it is unclear whether ileostomy or colostomy is superior. This study compared shortterm outcomes between palliative ileostomy and colostomy. Methods: We identified 82 patients with malignant large bowel obstruction, caused by various advanced cancers, between January 2005 and December 2016. We compared short-term outcomes between the ileostomy group $(\mathrm{n}=33)$ and the colostomy group $(\mathrm{n}$ = 49). Results: For all 82 patients, clinical success was achieved. Three patients with ileostomy died within 30 days of ostomy formation. The ileostomy group had statistically significant differences in median operative time (113 vs. 129 minutes, $p=0.045)$ and blood loss ( 8 vs. $40 \mathrm{~g}, p=0.037)$ in comparison with the colostomy group. No statistically significant differences were observed in the surgical complications (30.3 vs. $38.8 \%, p=0.431$ ), in the median period to oral intake ( 3 vs. 4 days, $p=0.335$ ) and in the hospital stay after surgery ( 32 vs. 27 days, $p=0.509$ ) between the two groups. Overall stoma-related complications occurred in 27 (32.9\%) patients. Stoma-related complications occurred more frequently in the ileostomy group (16/33 vs. $11 / 49$ patients, $p=0.014)$. High output stoma (6 patients) and irritation (5 patients) occurred more frequently in the ileostomy group. Conclusions: Palliative colostomy is superior to ileostomy due to fewer stoma-related complications. When ileostomy is required, aggressive interventions for high output stomas should be implemented.
\end{abstract}

Keywords:

palliative stoma, short-term outcome, colostomy, ileostomy

J Anus Rectum Colon 2019; 3(2): 73-77

\section{Introduction}

Multimodal therapy for patients with unresectable primary and recurrent malignant tumors prolongs survival times. However, malignant large bowel obstruction (MLBO) and fistula formation, such as rectovaginal fistula caused by malignant tumor invasion, impair quality of life (QOL).

Colonic metallic stent (CMS) placement is indicated for MLBO palliation because of its high success rate and low complication rate ${ }^{1-6)}$. However, CMS cannot be performed in patients with a high risk of clinical failure, including those with severe adhesions after laparotomy, bowel obstruction at multiple sites, and severe symptoms related to fistula formation and aggressive tumor invasion. In these patients, palliative interventions include stoma creation and bypass surgery.

Palliative ileostomies and colostomies are usually created at the sigmoid or transverse colon, but it is not clear that either type of ostomy is superior in terms of QOL.

The aim of this study was to compare short-term outcomes between palliative ileostomy and colostomy in patients with MLBO. 
Table 1. Ileostomy and Colostomy Patient Characteristics.

\begin{tabular}{|c|c|c|c|c|}
\hline & Total & Ileostomy group & Colostomy group & $\mathrm{p}$ value \\
\hline $\mathrm{N}$ & 82 & 33 & 49 & \\
\hline Age* (range) & $68.5(26-91)$ & $69(26-88)$ & $68(47-91)$ & 0.504 \\
\hline Gender & & & & 0.965 \\
\hline Male & $40(48.8)$ & $16(48.5)$ & $24(49.0)$ & \\
\hline Female & $42(51.2)$ & $17(51.5)$ & $25(51.0)$ & \\
\hline Primary disease & & & & 0.142 \\
\hline Colorectal & $44(53.6)$ & $16(48.5)$ & $28(57.2)$ & \\
\hline Gastric & $19(23.2)$ & $13(39.4)$ & $6(12.2)$ & \\
\hline Pancreatic and biliary ductal & $6(7.3)$ & $3(9.1)$ & $3(6.1)$ & \\
\hline Urological & $3(3.7)$ & 0 & $3(6.1)$ & \\
\hline Gynecological & $8(9.8)$ & $1(3.0)$ & $7(14.3)$ & \\
\hline Others & $2(2.4)$ & 0 & $2(4.1)$ & \\
\hline ASA PS status, grade & & & & 0.434 \\
\hline I & $5(6.1)$ & $2(6.1)$ & $3(6.1)$ & \\
\hline II & $64(78.0)$ & $24(72.7)$ & $40(81.6)$ & \\
\hline III & $13(15.9)$ & $7(21.2)$ & $6(12.2)$ & \\
\hline IV & 0 & 0 & 0 & \\
\hline Stoma site marking & & & & 0.309 \\
\hline Positive & $72(87.8)$ & $27(81.8)$ & 45 (91.8) & \\
\hline Negative & $10(12.2)$ & $6(18.2)$ & $4(8.2)$ & \\
\hline
\end{tabular}

( ): \%, *: median, ASA PS $=$ The American Society of Anesthesiologists physical status classification

\section{Method}

MLBO was defined as complete bowel obstruction, caused by a malignant tumor, that was diagnosed by computed tomography and required decompression.

This was a retrospective study of patients with MLBO who required stoma creation between January 2005 and December 2016 at Toho University Omori Medical Center.

We identified 82 patients with MLBO caused by unresectable primary or recurrent malignant tumor and evaluated the following factors: sex; age; cause of MLBO; presence of preoperative stoma site marking; surgical outcomes (operative time, blood loss, mortality, surgical and stoma-related complications, and hospital stay); and QOL (relief of obstructive symptoms, resumption of oral intake, period to oral intake, and clinical success). Surgical and stoma-related complications were scored according to the Clavien-Dindo (C-D) classification ${ }^{7}$. Clinical success was defined as the ability to pass flatus and stool after oral intake and relief of obstructive symptoms.

The study was approved by the ethics committee of Toho University Omori Medical Center (No. M18016). Also, informed consent was obtained from all study patients.

\section{Statistical Analysis}

The ileostomy group was compared with the colostomy group using the Chi square and Mann-Whitney U tests for categorical and continuous data, respectively. Continuous values were presented as medians (range). Statistical significance was considered as $\mathrm{p}<0.05$. All data analysis was performed using SPP statistics for Windows, version 22.0 (IBM SPSS, New York, USA).

\section{Results}

\section{Patient characteristics}

Patient characteristics are shown in Table 1. The study group consisted of 40 men and 42 women with a median age of 68.5 years (range, 26 years - 91 years). Malignant diseases leading to MLBO included colorectal cancer in 44 (53.6\%) patients, gastric cancer in $19(23.2 \%)$ patients, pancreatic and biliary ductal cancer in $6(7.3 \%)$ patients, urological cancer in $3(3.7 \%)$ patients, gynecological malignancies in $8(9.8 \%)$ patients, and other cancers in $2(2.4 \%)$ patients. Preoperative physical status included $5(6.1 \%)$ patients at American Society of Anaesthesiologists physical status (ASA-PS) classification grade I, 64 (78.0\%) at grade II, and $13(15.9 \%)$ at grade III. Preoperative stoma site marking was performed by nurses and/or surgeons in 72 $(87.8 \%)$ patients. There were no significant differences for the patients' characteristics between the two groups. 
Table 2. Comparison of Surgical Outcomes between Ileostomy and Colostomy.

\begin{tabular}{lccc}
\hline & Ileostomy group $(\mathrm{n}=33)$ & Colostomy group $(\mathrm{n}=49)$ & $\mathrm{p}$ value \\
\hline Operative time, minutes* (range) & $113(38-463)$ & $129(16-427)$ & 0.045 \\
Blood loss, g* (range) & $8(0-1350)$ & $40(0-1810)$ & 0.037 \\
Mortality (\%) & $3(9.1)$ & $30(61.2)$ & - \\
Surgical complications & & $19(38.8)$ & 0.431 \\
$\quad$ Negative & $23(69.7)$ & $15(30.6)$ & 0.092 \\
$\quad$ Positive (\%) & $10(30.3)$ & $4(8.2)$ & - \\
$\quad$ Grade I and II (\%) & $4(12.2)$ & $49(100)$ & - \\
$\quad 6(18.2)$ & $49(100)$ & 0.335 \\
Oral intake resumption (\%) & $33(100)$ & $4(1-16)$ & 0.509 \\
Obstructive symptoms improvement (\%) & $33(100)$ & $27(10-111)$ & \\
Period to oral intake, day* (range) & $3(1-61)$ & $32(10-151)$ &
\end{tabular}

Table 3. Comparison of Stoma-Related Complications between Ileostomy and Colostomy.

\begin{tabular}{|c|c|c|c|c|c|c|c|c|c|c|}
\hline & \multicolumn{5}{|c|}{ Ileostomy group $(\mathrm{n}=33)$} & \multicolumn{5}{|c|}{ Colostomy group $(n=49)$} \\
\hline & \multicolumn{4}{|c|}{ C-D classification } & \multicolumn{6}{|c|}{ C-D classification } \\
\hline & I & II & III & IV & Total $(\%)$ & I & II & III & IV & Total $(\%)$ \\
\hline Irritation & 3 & 2 & 0 & 0 & $5(15.2)$ & 1 & 2 & 0 & 0 & $3(6.1)$ \\
\hline Dehiscence & 2 & 0 & 0 & 0 & $2(6.1)$ & 4 & 1 & 0 & 0 & $5(10.2)$ \\
\hline High output & 1 & 5 & 0 & 0 & $6(18.2)$ & 0 & 2 & 0 & 0 & $2(4.1)$ \\
\hline Necrosis & 0 & 1 & 0 & 0 & $1(3.0)$ & 0 & 1 & 0 & 0 & $1(2.0)$ \\
\hline Prolapse & 0 & 0 & 1 & 0 & $1(3.0)$ & 0 & 0 & 0 & 0 & $0(0)$ \\
\hline Fistula & 0 & 1 & 0 & 0 & $1(3.0)$ & 0 & 0 & 0 & 0 & $0(0)$ \\
\hline Total & 6 & 9 & 1 & 0 & $16 *(48.5)$ & 5 & 6 & 0 & 0 & $11 *(22.4)$ \\
\hline
\end{tabular}

$*: \mathrm{p}=0.014, \mathrm{C}-\mathrm{D}$ classification $=$ the Clavien-Dindo classification

\section{Comparison of short-term outcomes between the ileostomy and colostomy groups}

\section{Surgical outcomes}

Surgical outcomes are shown in Table 2. The median operative time was significantly shorter in the ileostomy group than the colostomy group (113 minutes versus 129 minutes, $\mathrm{p}=0.045$ ). Median blood loss was significantly lower in the ileostomy group than the colostomy group ( $8 \mathrm{~g}$ versus $40 \mathrm{~g}$, $\mathrm{p}=0.037)$. Thirty-day mortality included $3(9.1 \%)$ patients in the ileostomy group. Surgical complications occurred in $29(35.4 \%)$ patients. There was no significant difference in the incidence of surgical complications between groups ( $\mathrm{p}=$ 0.431). Major surgical complications, defined as grade III or IV according to the C-D classification system, occurred in 6 (18.2\%) patients in the ileostomy group and 4 (8.2\%) patients in the colostomy group. This difference was not statistically significant $(\mathrm{p}=0.092)$. All patients resumed oral intake, and obstructive symptoms completely resolved. The median period to oral intake in the ileostomy group and the colostomy group were 3 days (range, 1 day-61 days) and 4 days (1 day-16 days), respectively. No statistically signifi- cant differences were observed between the two groups ( $\mathrm{p}=$ 0.335). The median duration of hospital stay was similar in both groups $(\mathrm{p}=0.509)$.

Stoma-related complications

Stoma-related complications occurred in 27 (32.9\%) patients. They occurred significantly more frequently in the ileostomy group than the colostomy group [16 patients $(48.5 \%)$ vs. 11 patients $(22.4 \%), p=0.014]$. High output stoma [6 patients $(18.2 \%)]$ and irritation [5 patients $(15.2 \%)$ ] occurred more frequently in the ileostomy group, while stoma dehiscence [5 patients, $(10.2 \%)$ ] occurred more frequently in the colostomy group (Table 3 ).

\section{Prognosis}

The median overall survival period of the ileostomy and colostomy groups were 86.5 days (range, 15 days -1515 days) and 157 days (range, 20 days -1597 days), respectively. After a median follow-up of 1345 days (range, 15 days -1597 days), overall survival rates were $9.1 \%$ in the ileostomy group and $0 \%$ in the colostomy group. There was no significant difference in overall survival between the two 


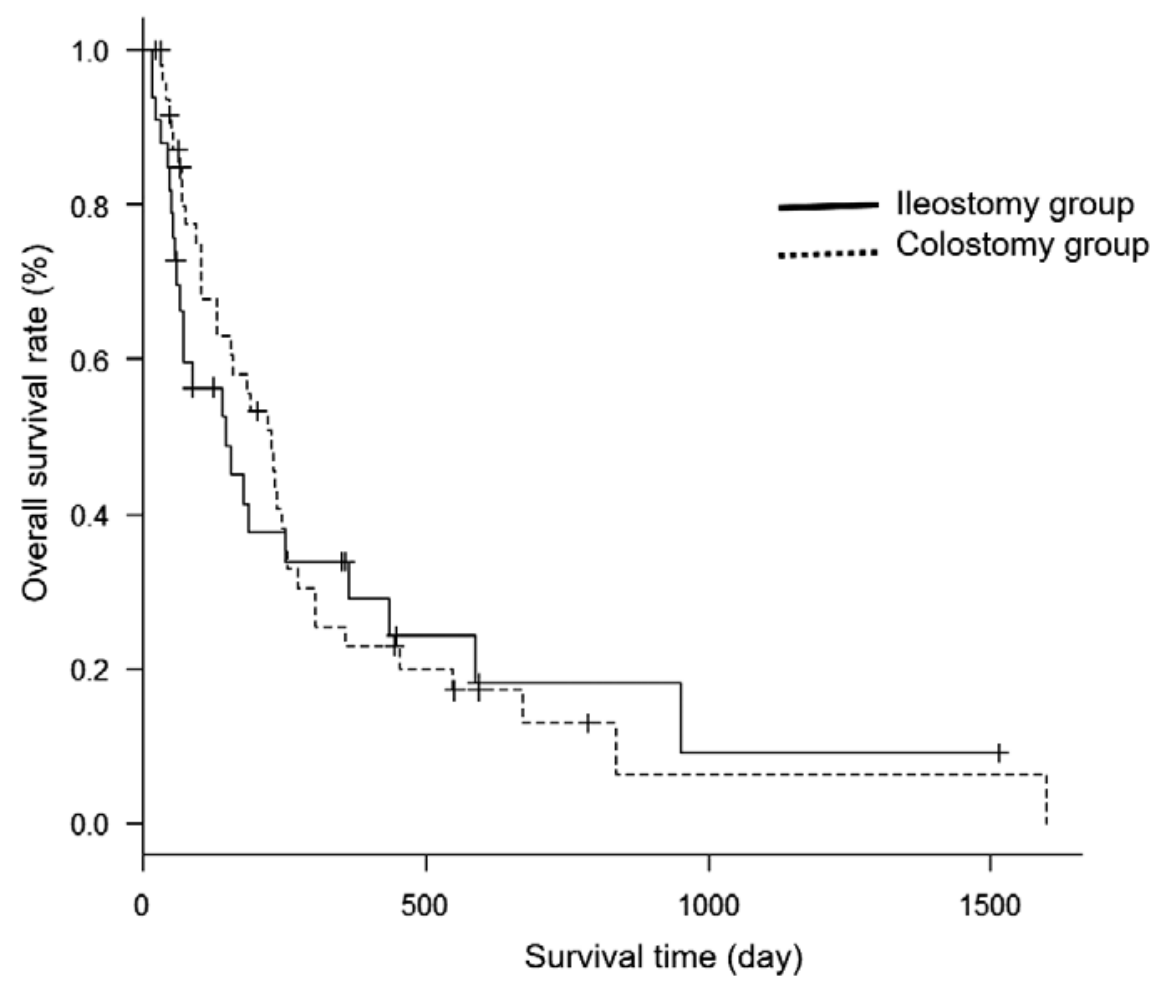

Figure 1. Overall survival rates in the ileostomy group and in the colostomy group. After a median follow-up of 1345 days (range, 15 days-1597 days), overall survival rates were $9.1 \%$ in the ileostomy group and $0 \%$ in the colostomy group. There was no significant difference in overall survival between the two groups $(\mathrm{p}=0.733)$.

groups $(\mathrm{p}=0.733$, Figure 1$)$.

\section{Discussion}

Bowel obstruction is a serious complication of several advanced malignant tumors, particularly pancreatic, gastric, and colorectal tumors, and peritoneal carcinomatosis of ovarian cancer. Bowel obstruction can significantly impair patient QOL. CMS is recommended as a therapeutic alternative to surgery, not only as a bridge to surgery but also for palliation. CMS leads to shorter duration of hospital stay, shorter time to initiation of chemotherapy, and lower rates of stoma formation compared with surgery. Conversely, palliative CMS is associated with a risk of short- and long-term complications such as perforation (0\%-8.9\%), stent migration $(4.4 \%-8 \%)$, restenosis $(4.4 \%-8 \%)$, and bleeding $(0 \%$ $3.7 \%)^{1,25,8-810)}$. Furthermore, clinical failure of CMS insertion is significantly associated with a higher 30-day mortality ${ }^{11}$.

For patients such as those with severe adhesions after laparotomy or aggressive tumor invasion into the abdominal cavity, CMS insertion is technically difficult. Palliative surgery might be safer and improve obstructive symptoms more reliably than CMS placement. Generally, when permanent stoma creation is required, colostomy is recommended because of benefits in stoma management and patient self-care compared with ileostomy. In this study, all 82 patients had a life expectancy of more than two months and good-tomoderate ASA-PS. Preoperative CT revealed obstruction at multiple sites or ascites ${ }^{12}$. In this study, the operator chose either ileostomy or colostomy based on the situation at the time. All 82 patients achieved clinical success and had symptom improvement with stoma creation. The overall surgical complication rate of $35.4 \%$ is comparable to that reported by Scheidbach et $\mathrm{al}^{13)}$, but the 30-day mortality included three patients in the ileostomy group. The three patients were in the terminal stage: one of them had cancer ascites due to recurrence of pancreatic cancer and the others had multiple liver metastases due to recurrence of colon cancer. For these patients with ascites and/or multiple liver metastases, palliative surgery CMS placement should have been performed ${ }^{12)}$.

A randomized clinical trial by Law et al. ${ }^{14)}$ showed that loop transverse colostomy for fecal diversion following low anterior resection with total mesorectal excision for rectal cancer caused few complications. Of four recent metaanalyses $^{15-18)}$, two recommended loop ileostomy for fecal diverting stoma after colorectal or coloanal anastomosis, and the other two found no differences between the two procedures. The authors concluded that high quality, large scale, randomized control studies were needed to obtain better evi- 
dence for making the decision between loop ileostomy or loop colostomy.

In this study, we compared short-term outcomes between ileostomy and colostomy to determine the superiority of one ostomy type for MLBO palliation. The ileostomy group had a significantly shorter operative time and significantly less blood loss than the colostomy group. The number of surgical complications was similar between the two groups. There was no significant difference in overall survival between the two groups. On the other hand, stoma-related complications, particularly major complications of C-D grade II or more, occurred more often in the ileostomy group than in the colostomy group. High output stoma was the most frequent major stoma-related complication in the ileostomy group. Stoma-related complications did not influence the duration of hospital stay. Patients with bowel obstruction tend to be dehydrated due to the accumulated of water and electrolytes in the intestine and poor fluid intake due to obstructive symptoms such as nausea and vomiting. While colonic stomas have the advantage of lower-volume, solid stools, and easier care after surgery, ileal stomas generally cause irritation, high output, and liquid stools ${ }^{17)}$. This study suggests that palliative colostomy is superior to ileostomy due to the latter's more frequent stoma-related complications related to high output.

However, this study has several limitations, includig its small sample size and single-center retrospective design. Additional randomized controlled trials are needed to clarify the superiority of either ileostomy or colostomy for palliation in terms of safety and QOL.

The decision to perform palliative surgery can be challenging because of the delicate balance between the pros and cons of intervention. The decision is influenced by the level of obstruction, clinical stage of the cancer, overall prognosis, presence of ascites, and patient performance status. When ileostomy is the only option, aggressive interventions for high output stomas should be implemented.

Conflicts of Interest

There are no conflicts of interest.

\section{References}

1. Sousa M, Pinho R, Proença L, et al. Predictors of complications and mortality in patients with self-expanding metallic stents for the palliation of malignant colonic obstruction. GE Port J Gastroenterol. 2017 May; 24(3): 122-8.

2. Finlayson A, Hulme-Moir M. Palliative colonic stenting: a safe alternative to surgery in stage IV colorectal cancer. ANZ J Surg. 2016 Oct; 86(10): 773-7.

3. Bayraktar B, Ozemir IA, Kefeli U, et al. Colorectal stenting for palliation and as a bridge to surgery: A 5-year follow-up study. World J Gastroenterol. 2015 Aug 21; 21(31): 9373-9.

4. Gianotti L, Tamini N, Nespoli L, et al. A prospective evaluation of short-term and long-term results from colonic stenting for pallia- tion or as a bridge to elective operation versus immediate surgery for large-bowel obstruction. Surg Endosc. 2013 Mar; 27(3): 83242.

5. Tominaga K, Maetani I, Sato K, et al. Favorable long-term clinical outcome of uncovered D-weave stent placement as definitive palliative treatment for malignant colorectal obstruction. Dis Colon Rectum. 2012 Sep; 55(9): 983-9.

6. Kim BC, Han KS, Hong CW, et al. Clinical outcomes of palliative self-expanding metallic stents in patients with malignant colorectal obstruction. J Dig Dis. 2012 May; 13(5): 258-66.

7. Clavien PA, Barkun J, de Oliveira ML, et al. The Clavien-Dindo classification of surgical complications: five-year experience. Ann Surg. 2009 Aug; 250(2): 187-96.

8. Gianotti L, Tamini N, Nespoli L, et al. A prospective evaluation of short-term and long-term results from colonic stenting for palliation or as a bridge to elective operation versus immediate surgery for large-bowel obstruction. Surg Endosc. 2013 Mar; 27(3): 83242.

9. Zhao XD, Cai BB, Cao RS, et al. Palliative treatment for incurable malignant colorectal obstructions: a meta-analysis. World J Gastroenterol. 2013 Sep 7; 19(33): 5565-74.

10. Liang TW, Sun Y, Wei YC, et al. Palliative treatment of malignant colorectal obstruction caused by advanced malignancy: a selfexpanding metallic stent or surgery? A system review and metaanalysis. Surg Today. 2014 Jan; 44(1): 22-33.

11. Kobborg M, Broholm M, Frostberg E, et al. Short-term results of self-expanding metal stents for acute malignant large bowel obstruction. Colorectal Dis. 2017 Oct; 19(10): O365-71.

12. Higashi H, Shida H, Ban K, et al. Factors affecting successful palliative surgery for malignant bowel obstruction due to peritoneal dissemination from colorectal cancer. Jpn J Clin Oncol. 2003 Jul; 33(7): 357-9.

13. Siddiqui A, Cosgrove N, Yan LH, et al. Long-term outcomes of palliative colonic stenting versus emergency surgery for acute proximal malignant colonic obstruction: a multicenter trial. Endosc Int Open. 2017 Apr; 5(4): E232-8.

14. Law WL, Chu KW, Choi HK. Randomized clinical trial comparing loop ileostomy and loop transverse colostomy for faecal diversion following total mesorectal excision. Br J Surg. 2002 Jun; 89 (6): 704-8.

15. Güenaga KF, Lustosa SA, Saad SS, et al. Ileostomy or colostomy for temporary decompression of colorectal anastomosis. Systematic review and meta-analysis. Acta Cir Bras. 2008 May-Jun; 23 (3): 294-303.

16. Rondelli F, Reboldi P, Rulli A, et al. Loop ileostomy versus loop colostomy for fecal diversion after colorectal or coloanal anastomosis: a meta-analysis. Int J Colorectal Dis. 2009 May; 24(5): 479-88.

17. Geng HZ, Nasier D, Liu B, et al. Meta-analysis of elective surgical complications related to defunctioning loop ileostomy compared with loop colostomy after low anterior resection for rectal carcinoma. Ann R Coll Surg Engl. 2015 Oct; 97(7): 494-501.

18. Chen J, Wang DR, Zhang JR, et al. Meta-analysis of temporary ileostomy versus colostomy for colorectal anastomoses. Acta Chir Belg. 2013 Sep-Oct; 113(5): 330-9.

Journal of the Anus, Rectum and Colon is an Open Access journal distributed under the Creative Commons Attribution-NonCommercial-NoDerivatives 4.0 International License. To view the details of this license, please visit (https://creativ ecommons.org/licenses/by-nc-nd/4.0/). 